\title{
ÜBER GESCHLOSSENE GEODÄTISCHE AUF GESCHLOSSENEN MANNIGFALTIGKEITEN
}

\author{
RYOJI SHIZUMA
}

\section{Einleitung}

In der vorliegenden Arbeit beschäftigen wir uns mit dem Problem der geschlossenen Geodätischen auf geschlossenen Mannigfaltigkeiten. Dieses Problem geht bekanntlich auf Morse $([4],[5])^{1)}$ zurück. Wir machen aber von seinem Kunstgriff des "zyklischen" Produktes [4] keinen Gebrauch; vielmehr kommt es uns hier darauf an, den Funktionalraum unseres Variationsproblems direkt in Betracht zu ziehen, wie es Morse beim analogen Problem der geodätischen Linien mit festen Randpunkte herausgearbeitet hat. Es wird sich im folgenden zeigen, wie sich sein Verfahren auf den Fall der geschlossenen Geodätischen übertragen lässt.

Begriffe und Bezeichnungen, die wir später benutzen, werden in $\S 1$ zusammengestellt.

Es wird sich dabei als zweckmässig erweisen, Funktionalraum unseres Variationsproblems als Faserbündel aufzufassen. In der Tat handelt es sich im folgenden nicht um den Raum $\Sigma$ aller geschlossenen Kurven, sondern um den Abbildungsraum $\widetilde{\Omega}$, die sich in natürlicher Weise Faserbündel über $\Sigma$ darstellt.

Nachdem die Grundbegriffe in $\S 1$ erklärt worden sind, spielt in $\S 2$ das Deformationsverfahren eine besondere Rolle. Die nun folgenden Überlegungen gehören demselben Ideenkreis an, der von Morse im oben gesagten Fall geklärt worden ist. Wir lehnen uns hier eng an das Buch von Seifert und Threlfall [6] an; jedoch entstehen dabei cinige Schwierigkeiten. Es ist wohl angebracht, den Begriff der Typenzahl der isolierten stationären Faser einzuführen.

Bezeichnet man mit $M^{k}$ die Summe der $k$-ten Typenzahlen aller isolierten stationären Fasern und mit $R^{k}$ die $k$-te Bettische Zahl von $\widetilde{\Omega}$ bis auf die Menge der konstanten Abbildungen, so lautet nun das Hauptresultat $(\S 3)$ :

Received February 21, 1958.

1) Die Nummern verweisen auf die Literatur am Schluss der Arbeit. 
Wenn es auf der Riemannschen Mannigfaltigkeit $R$ nur endlich viele geschlossene Geodätische von beschränkter Länge gibt, so ist $M^{k} \geq R^{k}$.

In $\S 4$ werden einige Anwendungen gezeigt. Wir erwähen unter anderem die folgende Tatsache:

Auf jeder geschlossen Mannigfaltigkeit $R$ gibt es wenigstens eine geschlossene Geodätische.

Besonders interessant erweist sich hier der Fall, wo $R$ einfach zusammenhängend ist. Im Fall, wo seine Fundamentalgruppe nicht verschwindet, ist die Behauptung übrigens schon bekannt [1] und leicht bewiesen.

\section{§1. Einige Funktionalräume}

Es sei $R$ eine $n$-dimensionale geschlossene Riemannsche Mannigfaltigkeit der Klasse $C^{3}$. Eine stetige Abbildung $f$ einer Strecke $I=[0,1]$ in $R$ heisst stückweise glatt, wenn es bis auf endlich vielen Punkten regulär ${ }^{2)}$ sind. Weiter rechnen wir die konstanten Abbildungen, die jeden Punkt in einen einzigen Punkt von $R$ abbildet, $z$ u den stückweise glatten.

Das stetige Bild einer Strecke heisst eine Kurve. Auf jeder stückweise glatten Kurve, die keine Punktkurve ist, werden wir stets die reduzierte Bogenlänge als Parameter benutzen. In dieser Weise entspricht einer solchen Kurve $C$ eine bestimmte Abbildung

$$
f: I \rightarrow R,
$$

die wir die zu $C$ gehörige kanonische Abbildung nennen.

Wir bezeichnen mit $J(C)$ die Länge einer Kurve und setzen ferner $J(f)$ $=J(C)$.

Die Riemannsche Mannigfaltigkeit $R$ ist bekanntlich dadurch metrisiert, dass man für zwei Punkte $x$ und $y$ auf $R$ die Entfernung $d(x, y)$ als die untere Grenze der Länge aller stückweise glatten Kurven von $x$ und $y$ definiert.

Wir betrachten zunächst Abbildungsraum $\Omega$ aller stetigen Abbildungen von einer orientierten Kreislinie $S^{1}$ auf $R$ :

$$
\Omega=\left\{f \mid f: S^{1} \rightarrow R\right\},
$$

2) Regulär bedeutet hier, wie üblich, differenzierbar mit nicht verschwindendem. Differentiale. 
mit der üblichen Topologie der gleichmässigen Konvergenz.

Wir. wählen nun einen festen Punkt $p$ auf $S^{1}$ und bilden $\Omega$ auf $R$ dadurch ab, dass wir jeder Abbildung $f$ von $\Omega$ den Punkt $f(p)$ von $R$ entsprechen lassen. Mit der so gewonnenen stetigen Abbildung

$$
\pi: \Omega \rightarrow R
$$

als Projektion erhält man den Faserraum im Serreschen Sinne [7], wie man leicht bestätigt.

Eine stückweise glatte Kurve $C$ ist, wie oben gesagt, von einer Abbildung

$$
f: I \rightarrow R
$$

definiert. Ist eine Kurve geschlossen, also $f(0)=f(1)=q$, so ergibt sich in natürlicher Weise eine ebenso mit $f$ bezeichnete Abbildung

$$
f: S^{1} \rightarrow R \quad \text { mit } f(p)=q
$$

wobei $p$ ein fester Punkt von $S^{1}$ ist.

Wir betrachten nun den Raum $\widetilde{\Omega}$ aller kanonischen Abbildungen von $S^{1}$ in $R$, die den stückweise glatten geschlossenen Kurven entsprechen. Die Entfernung zweier Abbildungen $f$ und $g$ von $\widetilde{\Omega}$ wird durch die Festsetzung

$$
d(f, g)=\operatorname{Max}_{t \in S^{1}} d(f(t), g(t))+|J(f)-J(g)|
$$

definiert, wobei $J(f)$ (bzw. $J(g)$ ) die Länge der Kurve $f\left(S^{1}\right)$ (bzw. $g\left(S^{1}\right)$ ) ist. Dadurch ist $\widetilde{\Omega}$ zum metrischen Raum geworden. Definitionsgemäss ist $J(f)$ eine stetige Abbildung von $f$.

Indem wir eine kanonische Abbildung als ein Element von $\Omega$ auffassen, so ergibt sich eine eineindeutig stetige Abbildung

$$
i: \widetilde{\Omega} \rightarrow \Omega
$$

Es sei $G$ die Rotationsgruppe von $S^{1}$, die mit $S^{1}$ homöomorph ist. Für $\sigma \in G$ und $f \in \widetilde{\Omega}$ setzt man

$$
\sigma f(t)=f(\sigma(t)), \quad t \in S^{1} .
$$

Man sieht leicht, dass einer stückweise glatten (orientierten) geschlossenen Kurve $C$ und einem Punkt $q$ auf $C$ eine eindeutig bestimmte Abbildung

$$
f:\left(S^{1}, p\right) \rightarrow(R, q)
$$

entspricht und dass umgekehrt jede of und nur diese die Kurve $C$ bestimmt. 
Der Quotientenraum $\hat{Q} / G$ werde mit $\Sigma$ bezeichnet. Dabei deuten wir $\Sigma$ als Raum aller stückweise glatten (orientierten) geschlossenen Kurven. $\widetilde{\Omega}^{3)}$ ist überdies in natürlicher Weise Prinzipal-Faserbündel über $\Sigma$ mit $G=S^{1}$ als Faser und Strukturgruppe [2].

In diesem Zusammenhang bemerken wir noch, dass die Länge $J(C)$ einer Kurve $C$ von $\Sigma$ wieder eine stetige Funktion auf $\Sigma$ ist.

\section{§ 2. Deformationssätze}

Unter einem stationären Faser in $\widetilde{\Omega}$ verstehen wir eine Faser, die einer geschlossenen Geodätischen auf $R$ entspricht. Unter einem stationären Werte verstehen wir einen Wert, den die Längenfunktion $J$ in einer stationären Faser annimmt, also die Länge einer geschlossenen Geodätischen. Wenn es zu einer stationären Faser $\mathfrak{F}$ eine Umgebung auf $\widetilde{\Omega}$ gibt, in der $\widetilde{F}$ die einzige stationäre Faser ist, so heisst $\mathfrak{\jmath}$ eine isolierte stationäre Faser auf $\hat{\Omega}$, und die entsprechende Geodätische auf $R$ heisst eine isolierte geschlossene Geodätische.

Ist $\alpha$ ein beliebiger Wert von $J$, so betrachten wir die Menge von $\widetilde{\Omega}$ :

$$
J_{\alpha}=\{f \mid J(f) \leqq \alpha\}
$$

bzw.

$$
J_{\alpha}^{-}=\{f \mid J(f)<\alpha\} .
$$

Die $k$-te Bettische Zahl von $J_{\alpha} \bmod J_{\alpha}^{-}$heisst die $k$-te Typenzahl des Wertes $\alpha$ :

$$
m^{k}=m^{k}(\alpha) \text {. }
$$

Hier und im folgenden benutzen wir immer die singuläre Homologietheorie und legen einen beliebigen Körper als Koeffizientenbereich zugrunde.

Ist $\widetilde{f}$ eine isolierte stationäre Faser mit $J(\hat{\jmath})=\alpha$, so heisst die $k$-te Bettische $Z$ ahl von $J_{\alpha}^{-} \cup \tilde{F} \bmod J_{\alpha}^{-}$die $k$-te Typenzahl einer stationären Faser $\tilde{i}: m^{k}(\mathfrak{i})$.

Es sei $U$ eine beliebige Umgebung von $\mathfrak{F}$, bezeichnen wir mit $U^{-}$derjenigen Punkt von $U$, für die $J<\alpha$ :

$$
U^{-}=U \cap J_{\alpha}^{-} .
$$

Man verifiziert leicht, dass die Bettische Zahlen von $U^{-} \cup \mathfrak{F} \bmod U^{-}$dieselben wie die von $J_{\alpha}^{-} \cup \mathfrak{F} \bmod J_{\alpha}^{-}$, also die Typenzahlen von $\tilde{F}$ sind.

3) Dabei muss man die konstanten Abbildungen ausschliessen. 
Wir beginnen mit dem folgenden

DeformationsSATz 1. Ist a nichtstationärer J-Wert, so gibt es eine J-Deformation $D$ von $J_{\alpha}$ in $J_{\alpha}^{-}$.

Unter einer $J$-Deformation versteht man folgendes: Bei einer Deformation nimmt der $J$.Wert eines jeden Punktes niemals zu.

Wir werden diese Deformation in zwei Schritten ausführen. Es sei $C$ eine stiickweise glatte geschlossene Kurve; ferner sei

$$
f: I \rightarrow R, \quad f(0)=f(1)=q
$$

irgendeine Abbildung aus der Faser über $C$.

Wir legen nun auf $C$ die $l$ Punkte fest, die den Parameterwerte

$$
t=0, \frac{1}{l}, \ldots, \quad \frac{l-1}{l}
$$

entsprechen und nennen sie

$$
q=q_{0}, q_{1}, \ldots, q_{l-1} .
$$

Sie teilen $C$ in $l$ gleich lange Teile. Wir wählen $l$ so gross, dass ${ }^{\alpha} l$ kleiner als die Elementarlänge $d$ von $R$ ist.

Wir bezeichnen mit $S(\tau, \sigma)$ die Sehne, die von $f(\tau)$ nach $f(\sigma)$ geht. Die Teilkurve von $C$, die man erhält, indem man die Parameter $t$ nur das Interval $\tau \leqq t \leqq \sigma$ durchlaufen lässt, wird mit $C(\tau, \sigma)$ bezeichnet.

Setzen wir nun

$$
\begin{aligned}
C=S\left(0, \frac{\tau}{l}\right)+C\left(\begin{array}{ll}
\tau & 1 \\
l & l
\end{array}\right)+\ldots & +S\left(\begin{array}{c}
l-1, l-1 \\
l
\end{array}\right) \\
& +C\left(\frac{l-1}{l}+\frac{\tau}{l}, 1\right), \quad 0 \leqq \tau \leqq 1,{ }^{11}
\end{aligned}
$$

so entspricht jeder Kurve $C_{\text {; }}$ die kanonische Abbildung

$$
f_{:}: l \rightarrow R, \quad f_{\mp}(0)=f_{-}(1)=q, \quad \text { wobei } f=f_{0} \text { ist. }
$$

Für $\tau=1$ ist $C=C_{0}$ in das geschlossene Elementarpolygon $C_{1}$ mit den Ecken $q_{i}$ übergegangen.

Wir wählen auf $C_{1}$ die $l$ Seitenmitten

4) Wenn der Endpunkt einer Kurve $A$ mit dem Anfangspunkt einer anderen Kurve $B$ zusammenfällt, so ist eine Kurve $A+B$ durch die Nacheinanderdurchläufung von $A$ und $B$ gegeben, 


$$
M_{1}, M_{2}, \ldots, M_{l}
$$

die in Bezug auf $f_{1}$ den Parameterwerte $t_{1}, t_{2}, \ldots, t_{q}$ entsprechen.

Nun setzen wir

$$
\begin{aligned}
C_{\tau} & =S\left((\tau-1) t_{1}, t_{1}\right)+S\left(t_{1}, t_{1}+(\tau-1)\left(t_{2}-t_{1}\right)\right) \\
& +C_{1}\left(t_{1}+(\tau-1)\left(t_{2}-t_{1}\right), t_{2}\right)+S\left(t_{2}, t_{2}+(\tau-1)\left(t_{3}-t_{2}\right)\right)+ \\
& +\cdot \cdot \cdot \cdot \cdot \cdot . \cdot . \\
& +S\left(t_{l-1}, t_{l-1}+(\tau-1)\left(t_{l}-t_{l-1}\right)+C_{1}\left(t_{l-1}+(\tau-1)\left(t_{l}-t_{l-1}\right), t_{l}\right)\right. \\
& +S\left(t_{l},(\tau-1) t_{1}\right) .
\end{aligned}
$$

Jeder Kurve $C \tau$ entspricht eindeutig die kanonische Abbildung

$$
f_{\tau}: I \rightarrow R, \quad 1 \leqq \tau \leqq 2
$$

mit $f_{\tau}(0)=f_{\tau}(1)=f_{1}\left((\tau-1) t_{1}\right)$.

Man überzeugt sich leicht davon, dass man durch die Festsetzung

$$
D(f, \tau)=f_{;}, \quad 0 \leqq \tau \leqq 2
$$

die gewünschte Deformation $D$ von $J_{\alpha}$ in $J_{\alpha}^{-}$erhält.

Damit ist der Satz bewiesen.

Aus der Konstruktion von $D$ gilt für jede Abbildung $f$

$$
J\left(f_{\tau}\right) \geq J\left(f_{\sigma}\right), \quad \tau \leqq \sigma .
$$

$D$ ist also eine $J$-Deformation und $J(f)=J\left(f_{2}\right)$ gilt dann und nur dann, wenn $f$ einer stationären Faser gehört.

Hilfssatz 1. Es sei $U$ eine offene Teilmenge von $J_{\alpha}$, die alle stationären Fasern enthält. Dann hat bei der Deformation D Verkürzung

$$
u(f)=J(f)-J\left(f_{2}\right)
$$

in $J_{\alpha}-U$ eine positive untere Grenze.

Man kann dies wörtlich wie bei [6] beweisen.

Es sei $C$ eine isolierte geschlossene Geodätische und

$$
f: I \rightarrow R, \quad f(0)=f(1)=q
$$

sei eine dazu gehörige kanonische Abbildung. Wir wählen auf $C l$ Punkte

$$
q=q_{0}, q_{1}, \ldots, \dot{q}_{l-1},
$$

und zwar so, dass die $l$ Teilbogen kürzer als die Elementarlänge sind. Wïr 
bestimmen durch $g_{i}(0 \leqq i \leqq l-1)$ eine Hyperfliche, die zweimal stetig differenzierbar in $R$ liegt und von $C$ in $q_{i}$ unter einem von Null verschiedenen Winkel durchsetzt wird. Auf jeder der Hyperflïchen wählen wir nun eine kleine Umgebung $V_{i}$ von $q_{i}$ von der Beschaffenheit, dass

1) $V_{i} \cap V_{i+1}=0$

2) je zwei Punkte aus $V_{i}$ und $V_{i+1}$ sich durch eine Elementarstrecke verbinden lassen,

3) diese Elementarstrecke nur ihre Randpunkte mit den beiden $V_{i}$ und $V_{i+1}$ gemeinsam hat.

Dabei ist $i \bmod l$ zu verstehen.

Wählt man in jeder der $l$ Umgebungen $V_{i}$ einen Punkt $r_{i}$ und verbindet aufeinanderfolgende Punkte $r_{i}$ und $r_{i \cdot 1}$ durch Elementarstrecken, so entsteht ein geschlossenes Elementarpolygon. Ferner fassen wir $r_{0} \in V_{0}$ als Anfangs-und Endpunkt auf.

Die sämtlichen Elementarpolygone, die man auf diese Weise erhält, bestimmen in $\widetilde{\Omega}$ eine mit $V_{0} \times V_{1} \times \ldots \times V_{l-1}$ homöomorphe Teilmenge $P$, die offenbar $f$ enthält.

Deformationssatz 2. Ist $W$ eine hinreichend kleine Umgebung von $f$ in $\widetilde{\Omega}$, so gibt es eine J-Deformation $\Delta$ von $W$, die $W$ เn $P$ so überführt, dass $W \cap P$ punktweise festbleibt.

Wir werden diese Deformation in drei Schritten ausführen.

$\mathrm{Zu}$ den $l$ Punkten $q_{0}, q_{1}, \ldots, q_{l-1}$ lassen sich Umgebungen in $R$

$$
U_{0}, U_{1}, \ldots, U_{l-1}
$$

von der Beschaffenheit wählen:

1) Sind $u_{0}, u_{1}, \ldots, u_{l-1}$ beliebige Punkte aus diesen Umgebungen, so bestimmen diese Punkte eindeutig ein geschlossenes Elementarpolygon.

2) Der Bogen zwischen $u_{i-1}$ und $u_{i+1}$ hat mit $V_{i}$ genau einen Punkt $r_{t}$ gemeinsam. $\quad r_{i}$ hängt stetig ab von $u_{i-1}, u_{i}, u_{i+1}$. Dabei ist $i \bmod l$ zu verstehen.

3) $r_{0}, r_{1}, \ldots, r_{l-1}$ bestimmen eindeutig ein geschlossenes Elementarpolygon.

$\mathrm{Zu}$ einem solchen Elementarpolygon entspricht eine eindeutig bestimmte kanonische Abbildung

$$
g: I \rightarrow R
$$


mit $g(0)=g(1)=\boldsymbol{r}_{11}$.

Es sei $h \in P$ beliebig gewählt. Ein dazu gehöriges Elementarpolygon habe $l$ Seiten mit den Längen $\lambda_{1}(h), \lambda_{2}(h), \ldots, \lambda_{l}(h)$. Es sei ferner $W^{\prime}$ eine Umgebung von $f$ in $\widetilde{\Omega} . \quad \lambda_{i}$ ist stetig und $>0$ auf $W^{\prime} \cap P$, lässt sich also auf $W^{\prime}$ erweitern, und zwar so, dass $\lambda_{i}>0$ ist.

Wir setzen

$$
t_{i}(k)=\frac{\lambda_{1}(k)+\ldots+\lambda_{i}(k)}{\lambda_{1}(k)+\ldots+\lambda_{l}(k)}, \quad k \in W^{\prime}
$$

und wählen auf $k(I) l$ Punkte $u_{0}(k), u_{1}(k), \ldots, u_{l-1}(k)$, die der Parameterwerte $0, t_{1}(k), \ldots, t_{l-1}(k)$ entsprechen. Wie man leicht ersieht, gibt es in $W^{\prime}$ eine so kleine Umgebung $W$ von $f$, dass jeder Punkt $u_{i}(k)$ in $U_{i}$ liegt, falls $k \mathrm{zu} W$ gehört, und dass jeder der Teilbogen $u_{i} u_{i+1}$ von $k$ kürzer als die Elementarlänge $d$ ist.

Wir wählen jetzt $k \in W$ beliebig, und bezeichnen mit $B$ die zu $k$ entsprechende Kurve. Setzen wir nun

$$
\begin{aligned}
B= & =S\left(0, \tau t_{1}(k)\right)+B\left(\tau t_{1}(k), t_{1}(k)\right)+ \\
& +\cdot \cdot \cdot \cdot \cdot\left(1-t_{l-1}(k) \tau\right) \\
& +S\left(i_{l-1}(k), t_{l-1}(k)+(k), \quad 0 \leqq \tau \leqq 1,\right.
\end{aligned}
$$

so geht $B=B_{0}$ für $\tau=1$ in das geschlossene Elementarpolygon $B$ mit den Ecken $u_{0}(k), u_{1}(k), \ldots, u_{l-1}(k)$ über.

Der Bogen von $B_{1} z$ wischen $u_{i-1}(k)$ und $u_{i+1}(k)$ hat mit $V_{i}$ einen Punkt $r_{i}(k)$ gemeinsam. Durch wiederholte Anwendung dieser Deformation geht $B_{1}$ in das geschlossene Elementarpolygon $B_{2}$ mit den Ecken $u_{0}(k), r_{0}(k), r_{1}(k), \ldots$, $r_{l-1}(k)$ bzw. $u_{0}(k), r_{2}(k), \ldots, r_{l-1}(k), r_{0}(k)$ über, je nachdem $r_{0}(k)$ auf dem Teilbogen, $u_{0}(k) u_{1}(k)$ bzw. auf $u_{l-1}(k) u_{0}(k)$ liegt.

Wir wenden nun auf $B$, die Deformation an, indem wir

bzw.

$$
B_{\tau+2}=S\left(\tau j \ell_{0}, \ell_{0}\right)+B_{2}\left(\mu_{0}, \mu l-1\right)+S\left(\mu l t-1, \tau \mu_{1}\right)
$$

$$
\begin{aligned}
= & S\left(\mu_{0}+(1-\tau)\left(1-\mu_{0}\right), \mu_{1}\right)+B_{2}\left(\mu_{1}, \mu_{0}\right) \\
& +S\left(\mu_{0}, \mu_{0}+(1-\tau)\left(1-\mu_{0}\right)\right) \quad(0 \leqq \tau \leqq 1)
\end{aligned}
$$

setzen, je nachdem $r_{0}(k)$ auf $u_{0}(k) u_{1}(k)$ bzw. auf $u_{l-1}(k) u_{0}(k)$ liegt. Dabei verstehen wir unter $\mu_{i}$ den reduzierten Parameter von $r_{i}(k)$ in Bezug auf den 
Anfangspunkt $u_{0}(k)$ auf $B_{2}$. Dadurch geht $B_{2}$ in das geschlossene Polygon $B_{3}$ mit den Ecken $r_{0}(k), r_{1}(k), \ldots, r_{l-1}(k)$ über.

Jeder Kurve $B$ - entspricht eindeutig die kanonische Abbildung

mit

$$
\begin{array}{rlrl}
k_{\tau}: I \rightarrow R, & 0 \leqq \tau \leqq 3 \\
k_{\tau}(0)=k_{\tau}(1) & =u_{0}(k), & 0 \leqq \tau \leqq 2 \\
& =k_{2}\left((\tau-2) \mu_{0}\right) & 2 \leqq \tau \leqq 3 \\
(\text { bzw. } & \left.=k_{2}\left(\iota_{0}+(3-\tau)\left(1-\mu_{0}\right)\right)\right) .
\end{array}
$$

Aus der Konstruktion folgt ohne weiteres, dass man durch die Festsetzung

$$
\Delta(k, \tau)=k_{;}, \quad 0 \leqq \tau \leqq 3
$$

die gesuchte Deformation $\Delta$ erhält.

\section{§3. Hauptsatz}

Es sei $g$ ein beliebiger Punkt von der isolierten stationären Faser i⿱ mit $J(\tilde{s})=\alpha . \quad W$ möge dieselbe Bedeutung haben wie im Deformationssatz 2.

Aus dem Deformationssatz 2 folgt wörtlich wie bei [6] der folgende

Hilfssatz 2. Es seien $U_{1}, U_{2}$ ein Paar von Umgebungen von g derart, dass

$$
W \supset U_{1} \supset \bar{U}_{2} \supset g
$$

gilt.

Dann gibt es eine J-Deformation $\varphi_{:}$von $\widetilde{\Omega}$, die $U_{2}$ in $J_{\alpha}^{-} \cup \widetilde{\&}$ überfïlurt und ausserhalb $U_{1}$ von $\widetilde{\Omega}$ punktweise festbleibt.

Wir fassen nun den Raum $\widetilde{\Omega}$ als Faserbündel über $\Sigma$ mit Projektion $\pi$ auf.

Wir setzen ferner $\pi(g)=C$ und bezeichnen mit $V(C)$ eine so kleine Umgebung von $C$, dass es ein fasertreuer Homöomorphismus

$$
\dot{\xi}: V(C) \times S^{1} \rightarrow \pi^{-1}(V(C))
$$

mit $\xi(C, \sigma)=g$ vorliegt.

Wir dürfen uns offenbar $\xi[V(C) \times V(\sigma)]$ als $U_{2}(g)$ bedienen, wobei $V(\sigma)$ eine kleine Umgebung von $\sigma$ ist. Weiter setzen wir

$$
\xi[V(C) \times \sigma]=V(g) .
$$

Aus $V(g) \subset U_{2}(g)$ gibt es nach dem obigen Satze eine Deformation $\hat{c}_{-}$, so dass für $\tau=1$ 


$$
\varphi_{1}: V(g) \rightarrow J_{\alpha}^{-} \cup \tilde{j}
$$

ist.

Durch die Festsetzung

$$
\psi=\left(C^{\prime}\right)=\pi \varphi_{-}\left(\xi\left(C^{\prime}, \sigma\right)\right), \quad C^{\prime} \in V(C)
$$

ist in $\Sigma$ eine Deformation $\psi_{\text {: von }} V(C)$ in $J_{\alpha}^{-} \cup C$ erklärt, da $J(f)=J(\pi(f))$ für $f \in \hat{\Omega}$ ist. Mit $\hat{J}_{\bar{\alpha}}^{-}$bezeichnet man dabei die Menge aller Punkte von $\Sigma$, in denen $J<\alpha$ ist.

Nach dem Satz von Überlagerung der Homotopie [3] hat man das kommutative Diagramm:

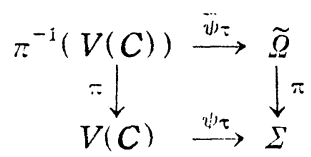

wobei $\bar{\psi}_{0}$ die identische Abbildung ist. $\bar{\psi}_{1}$ bildet daher $\pi^{-1}(V(C))$ in $J_{\alpha}^{-} \cup \tilde{f}$ ab. Wir wählen jetzt in $V(C)$ eine solche Umgebung $W(C)$ von $C$, dass $V(C) \supset \bar{W}(C)$ ist und konstruieren die stetige Funktion $\rho$ auf $\widetilde{\Omega}$, und zwar so, dass

$$
\begin{aligned}
& 0 \leqq \rho(f) \leqq 1 \\
& \rho(f)=1 \quad \text { auf } \quad \pi^{-1}(W(C)) \\
& =0 \quad \text { ausserhalb } \pi^{-1}(V(C)) \text {. }
\end{aligned}
$$

Setzt man

$$
\begin{aligned}
\widetilde{\psi}_{\tau}(f) & =\bar{\psi}_{\tau \cdot p(f)}(f), & & f \in \pi^{-1}(V(C)) \\
& =f & & \text { sonst, }
\end{aligned}
$$

so erhält man

SATz 1. Zu jeder isolierten stationären Faser $\widetilde{i}$ von $\widetilde{\Omega}$ gibt es ein Paar von $C_{\text {mgebungen }} U_{1}(\mathfrak{F}) \supset U_{2}(\tilde{\xi})$ von $\tilde{f}$ und eine J-Deformation $\tilde{\psi}_{\tau}$ von $\widetilde{\Omega}$ mit

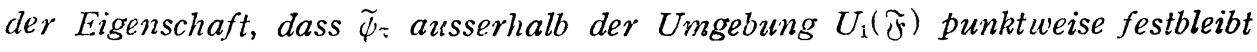
und $U_{2}(\mathfrak{F})$ in $J_{\alpha}^{-} \cup \mathfrak{F}$ ïberführt.

Wir machen jetzt die Voraussetzung, dass $\alpha$ ein nichtstationärer Wert ist und dass es nur endlich viele geschlossene Geodätische gibt, die kürzer als $\alpha$ sind. Es seien ferner: $\gamma$ der nächstkleinere stationäre Wert, $\hat{\vartheta}_{i}(i=1, \ldots, l)$ die Menge der stationären Fasern des Wertes $\gamma$.

$U_{1}\left(\mho_{i}\right), U_{2}\left(\widetilde{\mho}_{i}\right)$ habe dieselbe Bedeutung wie im Satz 1 . Die Umgebungen $U_{1}\left(\tilde{r}_{i}\right)$ von $\tilde{r}_{i}(i=1, \ldots, l)$ seien so gewählt, dass sie untereinander teilerfremd 
sind.

Nach dem Hilfssatze 1 sieht man leicht, dass $J_{\alpha}$ durch wiederholte Anwendung der Deformation $D$ in $J_{\sharp} \cup \bigcup_{i} U_{2}\left(\widetilde{J}_{i}\right)$ übergeführt wird.

Mit Hilfe der im Satz 1 genannten Deformationen geht $J_{x}$ schliesslich in $J_{\tilde{r}} \cup\left\{\tilde{\vartheta}_{i}\right\}$ über.

Damit haben wir den folgenden Satz bewiesen:

SATz 2. Ist $\alpha$ ein nichtstationärer Wert von $J$ auf $\widetilde{\Omega}$ und $\gamma$ der nächstkleinere stationäre Wert und gibt es auf $J_{\alpha}$ nur endlich viele stationäre Fasern, so lässt sich $J_{\alpha}$ durch eine J-Deformation überführen in $J_{\bar{Y}} \cup\left\{\widetilde{\mho}_{i}\right\}$, wobei $\tilde{F}_{i}$ stationäre Fasern des Wertes $r$ sind.

Aus dem Satz folgt ohne weiteres

Satz 3. Wenn es unterhalb jedes beliebigen J-Wertes nur endlich viele stationäre Fasern gibt, so ist die k-te Typenzahl $m^{k}(\gamma)$ des Wertes $\gamma$ gleich der Summe der k-ten Typenzahlen der einzelnen stationären Fasern $\tilde{f}_{1}, \mathfrak{f}_{2}, \ldots, \mathfrak{F}_{1}$ vom Werte $r$ :

$$
m^{k}(\gamma)=m^{k}\left(\widetilde{\mho}_{1}\right)+m^{k}\left(\mathfrak{\mho}_{2}\right)+\ldots+m^{k}\left(\mathfrak{\mho}_{l}\right)
$$

Hieran schliesst noch:

SATz 4. Wenn es auf $\widetilde{\Omega}$ unterhalb jedes beliebigen J-Wertes nur endlich viele stationäre Fasern gibt, so ist die folgende Bedingung erfüllt:

$Z u$ jedem nichthomologen relativen $Z y k l u s Z^{k}$ auf $\widetilde{\Omega} \bmod J_{0}=\{g \mid J(g)=0\}$, gibt es eine Zahl $\gamma$ derart, dass $Z^{k}$ homolog auf $\widetilde{\Omega}$ mod $J_{0}$ einem Zykel von $J_{r}$, aber nicht einem Zykel von $J_{r}^{-}$ist.

Der Beweis dieser Tatsache verläuft auf Grund des Satzes 2 ganz analog wie bei $[6]$.

Daraus und aus dem Satz $2([6] \S 5)$ folgt unmittelbar der folgende

HaUptsatz. Wenn es auf der Riemannschen Mannigfaltigkeit $R$ nur endlich viele geschlossene Gendätische von beschränkter Länge gibt, so ist

$$
M^{k} \geq R^{k}
$$

dabei bezoichnet $R^{k}$ die $k$-te Bettische Zahl von $\widetilde{\Omega}$ mod $J_{0}$, und $M^{k}$ die Summe der k-ten Typenzahlen aller isolierten stationären Fasern. 


\section{$\S$ 4. Einige Anwendungen}

Wir bemerken zuerst, dass $\ddot{\Omega}$ dom in $\S 1$ genannten Abbildungsraume $\Omega$ homologie-äquivalent ist.

Diese Tatsache kann man beweisen unter Benutzung der analogen Überlegungen wie bei $[6] \S 21$. Daraus folgt insbesondere, dass die Homologieeigenschaften von $\widetilde{\Omega}$ unabhängig von der Metrik auf $R$ sind.

Wir behaupten nun

SATz 5. Auf jeder geschlossenen Riemannschen Mannigfaltigkeit $R$ gibt es wenigstens eine geschlossene Geodätische.

Beweis. Wir fassen $\Omega$ als Faserraum über $R$ mit $F$ als Faser und mit Projektion $\pi: \Omega \rightarrow R$ auf.

Mit $r^{k}(R)$ bezeichenen wir wie üblich die $k$-te Homotopiegruppe von $R$.

Wir unterscheiden nun zwei Fälle.

1) $\pi_{1}(R)=0$

In diesem Fall ist $\Omega$ zusammenhängend. Wenn wir jedem Punkt $q$ von $R$ eine solche Abbildung entsprechen, die $S^{1}$ auf einen einzigen Punkt $q$ abbildet, so entsteht eine stetige Abbildung $\psi$ von $R$ in $\Omega$ mit $\pi \psi=$ Identität.

Hieraus folgt leicht unter Benutzung derselben Schlussweise wie im Fall des Faserbündels [6], dass

$$
\begin{aligned}
& \pi^{k}(\Omega)=\pi^{k}(R)+\pi^{k}(E), \quad k \geqslant 2 \\
& \pi^{1}(\Omega) / \pi^{1}(R)=\pi^{1}(E)
\end{aligned}
$$

ist.

Da andererseits immer

$$
\pi^{k}(E)=\pi^{k+1}(R)
$$

ist [7], ergibt sich nach Voraussetzung

$$
\begin{aligned}
& \pi^{k}(\Omega)=\pi^{k}(R)+\pi^{k+1}(R) \quad k \geqslant 2 \\
& \pi^{1}(\Omega)=\pi^{2}(R) .
\end{aligned}
$$

Infolge der Geschlossenheit von $R$ gibt es eine ganze Zahl $r$ derart:

$$
\begin{array}{ll}
\pi^{k}(R)=0, & 0 \leqq k<r \\
\pi^{r}(R) \neq 0, & 1<r \leqq n .
\end{array}
$$

Daraus folgt unmittelbar: 


$$
\begin{aligned}
& \pi^{k}(\Omega)=0 \\
& \pi^{r-1}(\Omega) \neq 0 .
\end{aligned}
$$

Nach dern Hurewiczschen Satz ist

$$
H_{*}^{r-1}(\Omega)=\pi^{r-1}(\Omega),
$$

unter $H_{*}^{r-1}(\Omega)$ die ganzzahlige Homologiegruppe verstanden. Bei geeigneter Wahl von Koeffizientenkörper dürfen wir annehmen, dass die $r-1$-te Homologiegruppe von $\Omega$ nicht verschwindet, also dass $R^{r-1}(\Omega) \neq 0$ ist.

Aus dem vorigen Bemerkung folgt nun

$$
R^{r-1}(\widetilde{\Omega})=R^{r-1}(\Omega) .
$$

Da $H_{*}^{r-1}\left(J_{0}\right)=H_{*}^{r-1}(R)=0$, folglich $H_{*}^{r-1}\left(\widetilde{\Omega}, J_{0}\right)=H_{*}^{r-1}(\widetilde{\Omega})$ ist, ist $R^{r-1}\left(\widetilde{\Omega}, J_{0}\right)$ $=R^{r-1}(\widetilde{\Omega})$.

Daraus folgt nach Hauptsatz

$$
M^{r-1}(\widetilde{\Omega})>0, \quad r-1 \geq 1 .
$$

Daher gibt es eine Zahl $\gamma>0$ derart, dass $m^{r-1}(\gamma) \neq 0$ ist.

Damit ist der erste Fall erledigt.

2) $\pi^{1}(R) \neq 0([1]$ Theorem 5.4)

In diesem Fall zerfällt $Q$ in seine disjunkte Komponenten, von denen jede zusammenhängend ist und einer der freien Homotopieklassen der Abbildungen von $S^{1}$ in $R$ entspricht. Es sei

$$
\varphi:\left(S^{1}, p\right) \rightarrow(R, q)
$$

eine solche Abbildung, die ein nichttriviales Element von $\pi^{1}(R)$ darstellt. Man darf von vornherein annehmen, dass $\varphi$ eine kanonische Abbildung ist, m.a.W. bei der in $\S 1$ genannten Injektion $i: \widetilde{\Omega} \rightarrow \Omega i(f)=\xi$ ist, wobei $f$ die mit $\varsigma$ identische Abbildung ist.

Ferner sei $J(f)=\alpha$. Im Gegensatz zu der Behauptung nehmen wir jetzt an, dass es auf $R$ keine Geodätische gibt. Durch wiederholte Anwendung der Deformation $D$ sicht man leicht, dass $f$ in $J_{0}=\{g \mid J(g)=0\}$ übergeführt wird. Daraus folgt unter Benutzung der Injektion $i$, dass $\varphi$ in $\Omega$ frei homotop Null, also schlechthin homotop Null ist. Dies ist aber im Widerspruch mit der Wahl von $\hat{c}$.

Damit ist der Satz vollständig bewiesen. 
Aus dem obigen Beweise geht ohne weiteres hervor, dass sich der Satz 5 folgendermassen verfeinern lässt: Es sei $s$ die Anzahl der freien Homotopieklassen der Abbildungen von $S^{1}$ in $R$. Dann gibt es wenigstens s geschlossenen Geodätischen auf $R$.

\section{LITERATUR}

[1] S. S. Chern, Lecture note on differential geometry, 1952.

[2] A. M. Gleason, Spaces with a compact Lie group of transformations, Proc. Amer. Math. Soc., 48 (1950), pp. 35-43.

[3] W. Huebsch, On the covering homotopy theorem, Ann. of Math., 61 (1955), pp. 555563.

[4] M. Morse, The calculus of variations in the large, 1934.

[5] M. Morse, Function topology and abstract variational theory, 1938.

[6] H. Seifert und Threlfall, Variationsrechnung im grossen, 1939.

[7] J.-P. Serre, Homologie singulière des espaces fibrés, Ann. of Math., 54 (1951), pp. 425505.

[8] N. Steenrod, Topology of fiber bundles, 1951.

Nagoya, Mathematisches Institut der Universität 